\title{
Additions to the Limoniidae and Pediciidae fauna of Morocco, with an updated checklist (Diptera, Tipuloidea)
}

\author{
Ouafaa Driauach', Boutaïna Belqat ${ }^{\prime}$ \\ I Laboratory «Ecology, Biodiversity and Environment», Department of Biology, Faculty of Sciences, University \\ Abdelmalek Essaâdi, Tétouan, 93030, Morocco \\ Corresponding author: Ouafaa Driauach (driauach@gmail.com)
}

Academic editor: J. Salmela | Received 2 December 2015 | Accepted 20 January 2016 | Published 15 February 2016

http://zoobank.org/F408D3D4-OFBD-4841-9178-5A21EBDF6770

Citation: Driauach O, Belqat B (2016) Additions to the Limoniidae and Pediciidae fauna of Morocco, with an updated checklist (Diptera, Tipuloidea). ZooKeys 563: 129-146. doi: 10.3897/zookeys.563.7384

\begin{abstract}
Eighteen species of Limoniidae and two species of Pediciidae are recorded for the first time in Morocco, of which 15 species are new to North Africa. An updated checklist of Moroccan short-palped craneflies (Limoniidae and Pediciidae) is appended, containing 73 species in 25 genera.
\end{abstract}

\section{Keywords}

Morocco, new records, North Africa, Short-palped crane flies, updated checklist

\section{Introduction}

Limoniidae and Pediciidae belong to the Tipuloidea together with Cylindrotomidae and Tipulidae. The Limoniidae comprise more than 741 species already known in the Westpalaeartic region, from which only 102 species are known to occur in Morocco (49), Algeria (32), Tunisia (9), Libye (1) and Egypt (11). The Pediciidae are the second smallest family of the Tipuloidea, with 77 recognized taxa in the Westpalaeartic of which only 4 were recorded from North Africa, in Morocco (Driauach et al. 2013, Oosterbroek 2015). 
Adults of Limoniidae and Pediciidae are associated with moist environments. They are usually collected from damp vegetation along the borders of various water bodies and from damp forests (Starý et al. 2005, De Jong et al. 2008). Their immature stages are usually found in a wide range of aquatic and semi-aquatic habitats (Reusch and Oosterbroek 1997).

Limoniidae and Pediciidae are poorly known in Morocco. They only received sporadic faunistic records made by European entomologists (Pierre 1922a, b, Pierre 1924, Séguy 1941a, b, Vaillant 1956, Starý 1971, Starý 1974, Savchenko et al. 1992, Eiroa 2000, Pârvu et al. 2006, Starý 2006, Starý and Oosterbroek 2008, Starý 2009, Starý 2011).

Only a single attempt was made to summarize the knowledge of the Moroccan fauna in the first checklist of the short-palped crane flies of Morocco (Driauach et al. 2013) and very recent research also revealed a new species, Baeoura staryi from Morocco including an identification key of the genus Baeoura in the West Palaearctic species (Driauach and Belqat 2015).

Prior to the present study, there were 53 species recorded from Morocco (Oosterbroek 2015). New findings increase the number of Moroccan Limoniidae and Pediciidae to 73. Altogether 44 species of Limoniidae and Pediciidae are collected and recorded in this work. Of these, 18 species of Limoniidae and two species of Pediciidae are recorded for the first time in Morocco of which 15 species are new to North Africa.

\section{Material and methods}

The material presented in this work come from field campaigns carried out by the authors between 2011 and 2015 in 77 sites distributed over a mountainous area (Rif, High Atlas, Middle Atlas, Beni Snassen) and arid area (Sahara) of Morocco. A total of 564 specimens of short-palped crane flies (515 specimens of Limoniidae and 49 specimens of Pediciidae) were identified. Adults were mostly collected by sweeping vegetation with entomological hand net whereas other specimens were reared in the laboratory from the substratum taken from several zones of water bodies margins and kept under the laboratory conditions. Fresh material was stored in $70 \%$ alcohol. For identification, the male terminalia, if necessary, were prepared by boiling in a solution of $10 \% \mathrm{KOH}$ and preserved in glycerine. All the specimens were collected by the authors and are deposited in the Diptera Collection of the Faculty of Sciences, University Abdelmalek Essaâdi, Tétouan, Morocco. The Distribution as it is given for individual species is based on Oosterbroek (2015).

The checklist of Moroccan short-palped craneflies is updated. New records are marked with an asterisk $\left(^{*}\right)$ and bold set text. A brief description of the material examined is given and the distribution of the identified species is provided, together with notes on the breeding sites (Table 1). 


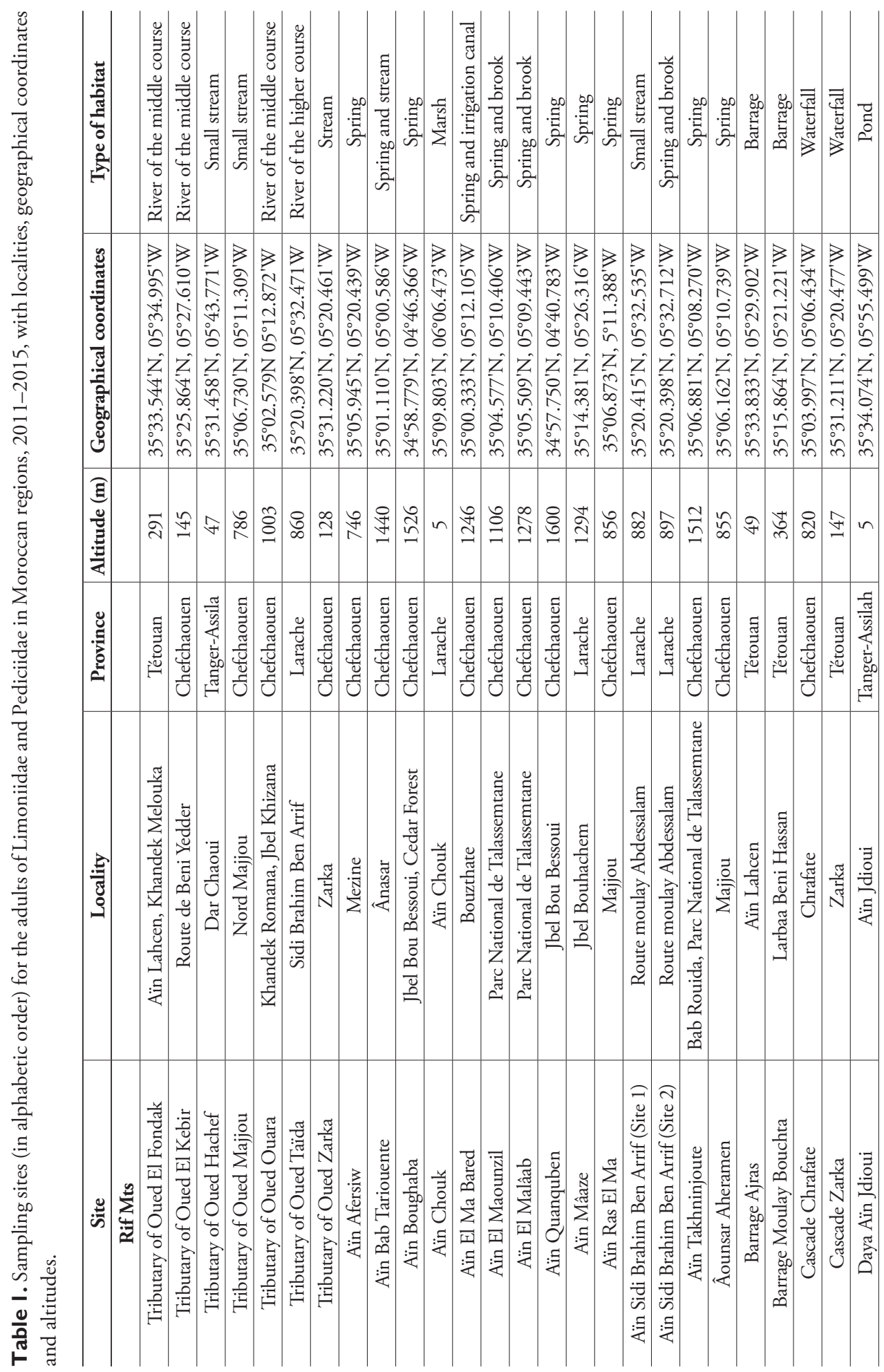




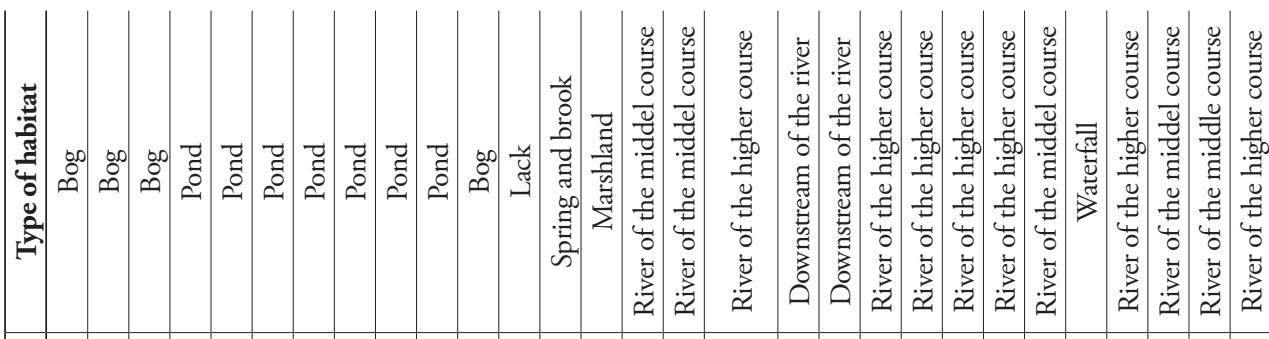

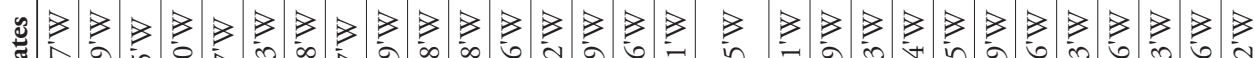

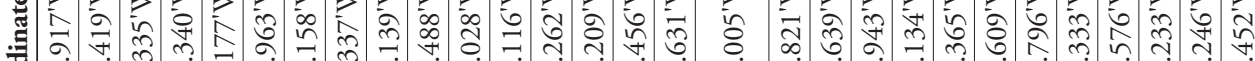

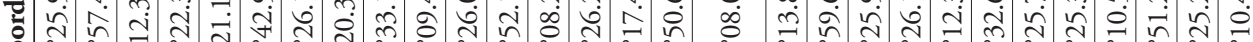

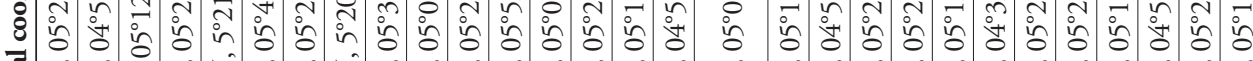
త⿹\zh26灬

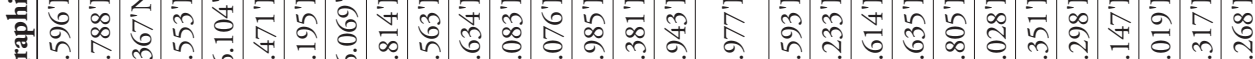

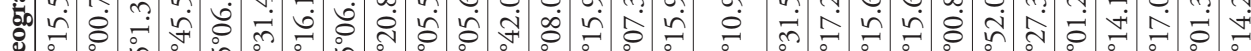

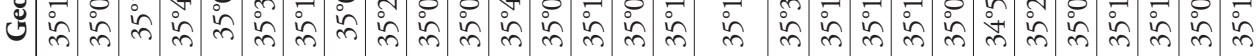

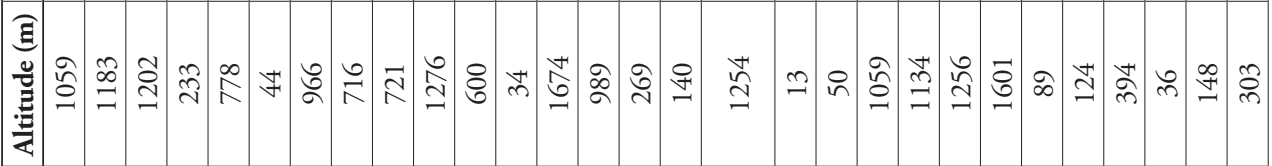

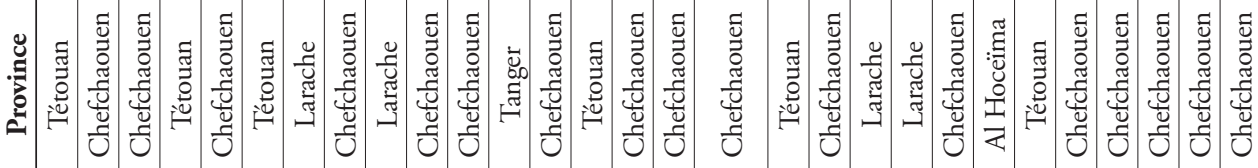

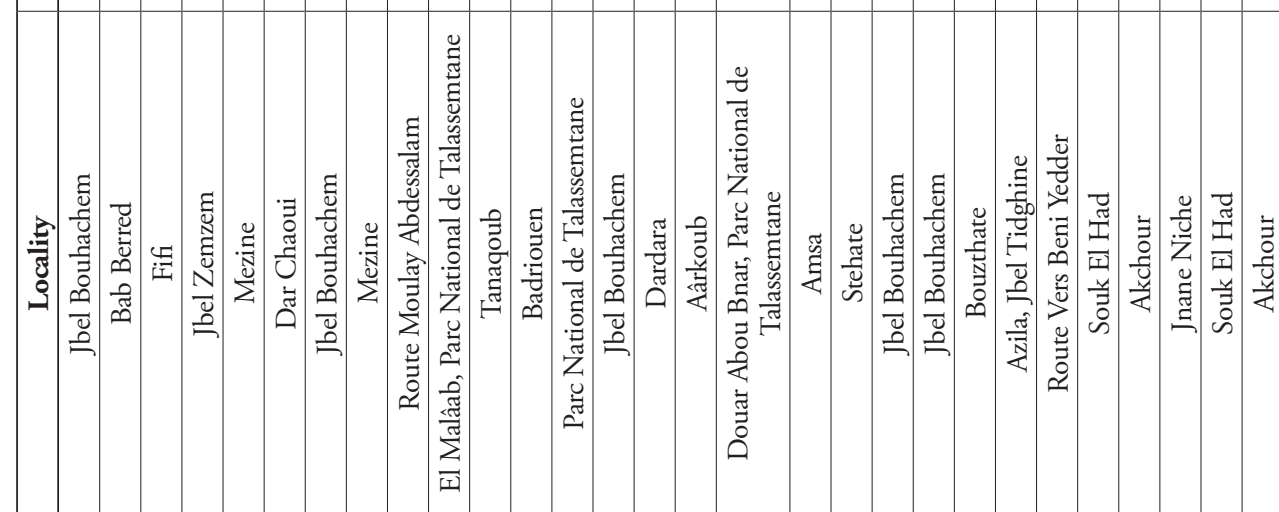




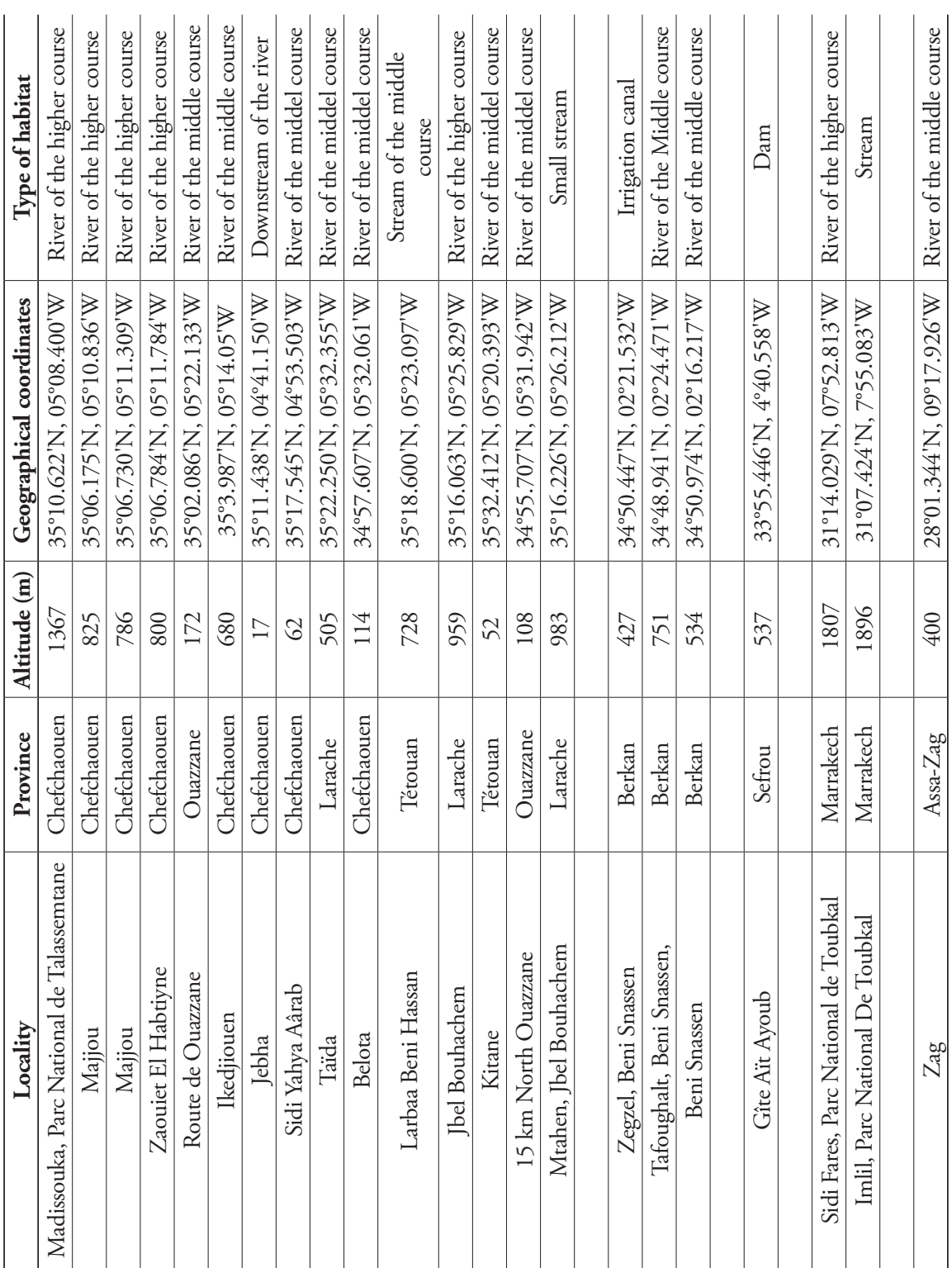

๑ี

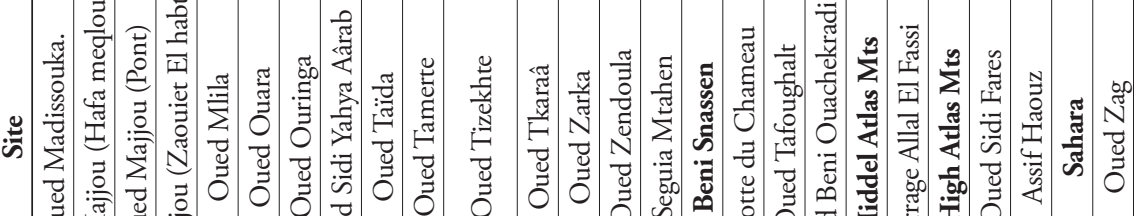

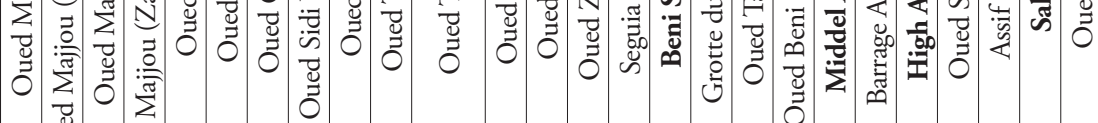




\section{Systematic part}

\section{Limoniidae: Chioneinae}

Cheilotrichia (Empeda) cinerascens (Meigen, 1804)

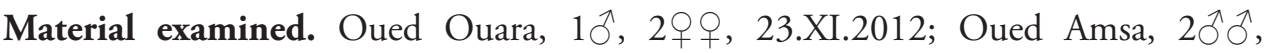
13.XII.2013; Oued Majjou (Hafa meqlouba), 10, 10.V.2014; Grotte du Chameau,

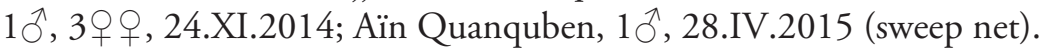

Distribution. Europe, Morocco, Georgia, Turkey, Cyprus, Israel, Iran. First record from Beni Snassen.

\section{Cheilotrichia (Empeda) fuscohalterata (Strobl, 1906)}

Material examined. Daya Fifi, $1 \widehat{\partial}, 23 . X I .2012$; Tributary of Oued El Fondak, $2 \hat{\partial}$, 10.II.2013, 1ð, 10.IV.2013; Barrage Ajras, $2 \widehat{\jmath}$, 10.II.2013; Daya Mghara, $6 \widehat{\jmath}$,

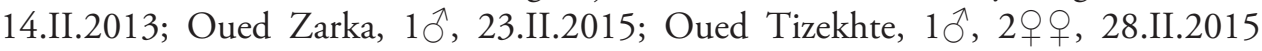
(sweep net).

Distribution. Italy, Portugal, Spain, First records from Morocco and North Africa.

Cheilotrichia (Empeda) minima (Strobl, 1898)

Material examined. Oued Zendoula, $2 \hat{\jmath} \widehat{\partial}, 31 . V .2013$; Oued Jnane Niche, $1 \hat{\partial}, 1$, 25.IV.2015 (sweep net).

Distribution. Known from Europe (except for the north) and the south of the Palaearctic as far east as Kyrgyzstan. First records from Morocco and North Africa.

\section{Ellipteroides (Ellipteroides) lateralis (Macquart, 1835)}

Material examined. Daya Afrate, $3 \hat{\partial} \widehat{\partial}$, 18.IV.2015 (sweep net).

Distribution. Southern part of Europe, Morocco, Lebanon, Israel and Turkey. First record from the Rif.

\section{Erioconopa diuturna (Walker, 1848)}

Material examined. Daya Jbel Zemzem, 1 $\hat{\sigma}$, 23.IV.2014; Oued Mezine, $4 \hat{\jmath} \widehat{\partial}$, 18.IV.2015; Aïn Bab Tariouente, 2ð̂̋, 2웅, 28.IV.2015 (sweep net).

Distribution. Europe, Morocco, Turkey. First record from the Rif. 


\section{Erioptera (Erioptera) fuscipennis Meigen, 1818}

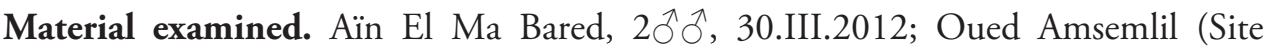

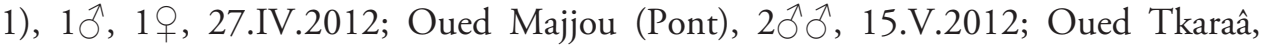

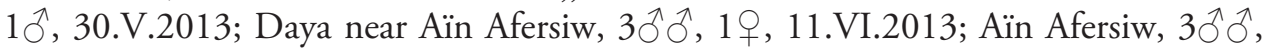

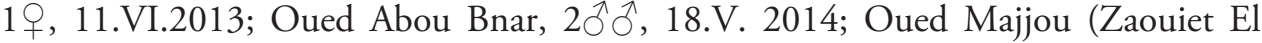

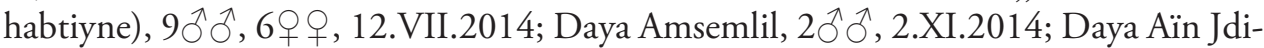

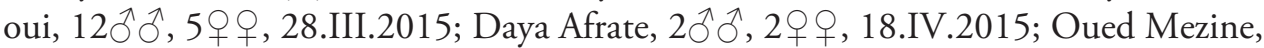
1ठ, 18.IV.2015 (sweep net).

Distribution. Europe, Morocco, Algeria, Azerbaijan, Iran.

\section{Erioptera (Erioptera) lutea lutea Meigen, 1804}

Material examined. Aïn Boughaba, 1§̂, 24.V.2013 (sweep net).

Distribution. Europe, Transcaucasia, Turkey, Israel, ?Iran, Russia, Kazakhstan, Uzbekistan, Tajikistan, Kyrgyzstan. First records from Morocco and North Africa.

\section{Gonomyia (Gonomyia) abscondita Lackschewitz, 1935}

Material examined. Maison forestière, $3 \hat{\jmath} \widehat{\jmath}$, 21.IV.2015 (sweep net).

Distribution. Austria, Bulgaria, Czech Rep., Finland, Germany, Great Britain, Italy, Netherlands, Romania, Slovakia, Sweden, Ukraine. First records from Morocco and North Africa.

\section{Gonomyia (Gonomyia) subtenella (Meigen, 1818),}

Material examined. Oued Beni Ouachekradi, $2 \widehat{\partial}$, 30.XII.2014, 1ㅇ, 8.I.2015 (reared); Oued Jnane Niche, 1§, 25.IV.2015; Oued Sidi Yahya Aârab, 1§, $3 q$, 25.IV.2015 (sweep net).

Distribution. Czech Rep., Lithuania, Macedonia, Slovakia, Sweden, Morocco (High Atlas), Georgia, Azerbaijan, Iran. First records from Rif and Beni Snassen.

\section{Gonomyia (Gonomyia) sicula Lackschewitz, 1940}

Material examined. Oued El Kebir, 1ڤึ, 17.IV.2013; Daya Jbel Zemzem, 7ðへ, 23.IV.2014; Aïn Sidi Brahim Ben Arrif (Site 2), 10, 25.IV.2014; Daya Tazia, 10, 25.IV.2014; Aïn El Maounzil, 1ठ, 21.IV.2015 (sweep net).

Distribution. Italy, Spain, Tunisia, Turkey. First records from Morocco. 
Hoplolabis (Parilisia) sororcula (Lackschewitz, 1940)

Material examined. Barrage Ajras, $3 \hat{\jmath}, 1$, 9 , 10.II.2013; Oued Ouringa, $1 \hat{\partial}$,

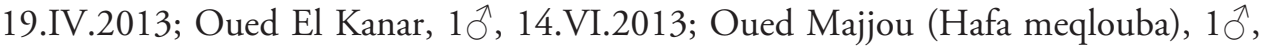
10.V.2014 (sweep net).

Distribution. France, Germany, Italy (incl. Sicily), Portugal, Romania, Slovakia, Spain, Switzerland, Ukraine, Morocco. First records from the Rif.

\section{Idiocera (Idiocera) ampullifera (Stary, 1979)}

Material examined. Oued Zag, 1ठ, 1, 22.V.2015 (sweep net).

Distribution. Egypt, Israel. First record from Morocco.

\section{Idiocera (Idiocera) pulchripennis (Loew, 1856)}

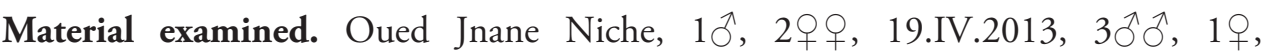

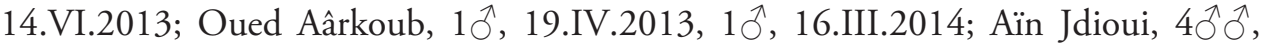
2우우, 28.III.2015 (sweep net).

Distribution. Southern part of Europe, Canary Islands, Morocco, Algeria, Egypt, Transcaucasia, Turkey, Cyprus, Israel, Iran, Central Asia. First record from the Rif.

Idiocera (Idiocera) sziladyi (Lackschewitz, 1940)

Material examined. Oued Zarka, 1ð, 22.V.2014 (light trap).

Distribution. Europe (except for northern countries), Algeria, Egypt, Yemen. First record from Morocco.

\section{Ilisia maculata (Meigen, 1804)}

Material examined. Oued Tafoughalt, 2 $\widehat{\partial}$, 25.XI.2014; Oued Majjou (Hafa meqlouba), 10̂, 27.IV.2015 (sweep net).

Distribution. Europe, Transcaucasia, Turkey, Iran. First records from Morocco and North Africa.

\section{Molophilus (Molophilus) obscurus (Meigen, 1818)}

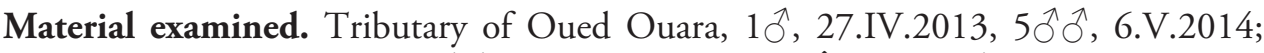

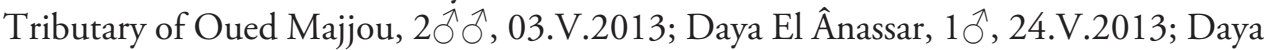

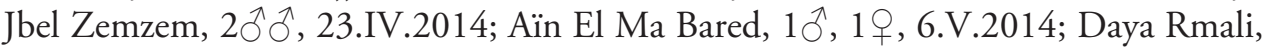


10, 17.V.2014; Oued Amsemlil (Site 1), 10ิ, 2.XI.2014; Aïn Mâaze, 10, 1.XI.2014; Daya Afrate, $9 \hat{\jmath}$, 18.IV.2015; Daya Mtahen, 10̂, 25.V.2015 (sweep net).

Distribution. Europe, Morocco (High Atlas), Transcaucasia, Turkey, Cyprus, Lebanon, Israel. First records from the Rif.

Molophilus (Molophilus) propinquus propinquus (Egger, 1863)

Material examined. Oued Ouara, 1 $\hat{\partial}$, 7.I.2013 (reared); Oued Farda, $3 \hat{\partial} \hat{\partial}$, 24.IV.2013; Tributary of Oued Taïda, 10ิ, 25.IV.2014; Oued Majjou (Hafa meqlouba), 10, 27.IV.2015 (sweep net).

Distribution. Europe, Morocco (High Atlas), Georgia, Turkey. First records from the Rif.

\section{Molophilus (Molophilus) testaceus Lackschewitz, 1940}

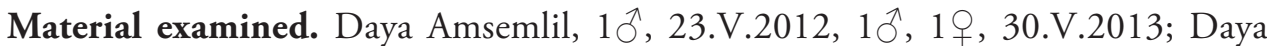

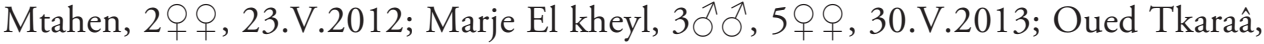

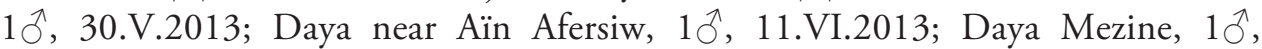
11.VI.2013; Daya Tazia, 1ð, 25.IV.2014; Daya Rmali, 1ठ, 17.V.2014 (sweep

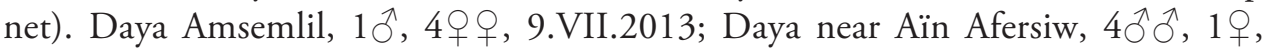
2.VII.2013 (reared).

Distribution. Only known from Portugal and Spain. First records from Morocco and North Africa.

\section{Symplecta (Symplecta) bybrida (Meigen, 1804)}

Material examined. Barrage Ajras, 10, 10.II.2013; Barrage Allal El Fassi, $1 \hat{\partial}$, 15.IV.2014; Oued El Kanar, 19, 14.VI.2013, 10, 25.IV.2015; Oued Aârkoub, 10̄, 16.III.2014; Oued Jnane Niche, 19, 25.IV.2015 (sweep net).

Distribution. Nearctic (Canada, USA, Greenland); widespread in Palaearctic, including North Africa, Central Asia, Mongolia, as far east as North Korea and Japan; Oriental (India, Nepal, Pakistan). First records from the Rif and the Middle Atlas.

\section{Symplecta (Trimicra) pilipes (Fabricius, 1787)}

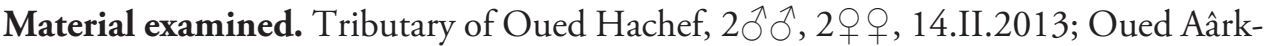

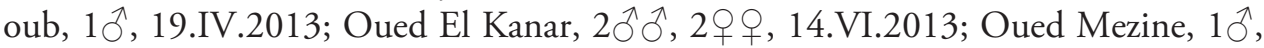
18.IV.2015 (sweep net); Aïn Chouk, 1ठ, 3.II.2015 (reared).

Distribution. The only cosmopolitan cranefly, widespread in all zoogeographical regions. First records from the Rif. 
Tasiocera (Dasymolophilus) murina (Meigen, 1818)

Material examined. Oued Farda, 1 $\widehat{\partial}$, 28.III.2013; Oued Amsemlil (Site 3), $4 \hat{\jmath} \widehat{\partial}$, 30.V.2013 (sweep net).

Distribution. Europe, Transcaucasia, Turkey. First records from Morocco and North Africa.

\section{Limoniidae: Limnophilinae}

Austrolimnophila (Austrolimnophila) latistyla Stary, 1977

Material examined. Oued Majjou (Hafa meqlouba), 1ð, 27.VI.2015 (sweep net).

Distribution. Croatia, France, Greece, Italy, Portugal, Spain. First record from Morocco and North Africa.

\section{Dicranophragma (Brachylimnophila) nemorale (Meigen, 1818)}

Material examined. Aïn Sidi Brahim Ben Arrif (Site 1), 10, 25.IV.2014; Tributary Oued Ouara, 2우, 27.IV.2013; Oued Majjou (Hafa meqlouba), 10ิ, 10.V.2014 (sweep net).

Distribution. Widespread in the Palaearctic. First records from the Rif.

\section{Euphylidorea crocotula (Seguy, 1941)}

Material examined. Aïn Bab Tariouente, $3 \hat{\jmath} \widehat{\partial}$, 28.IV.2015 (sweep net).

Distribution. Spain (Granada), Morocco (High Atlas). First record from the Rif.

Euphylidorea (Euphylidorea) dispar (Meigen, 1818)

Material examined. Oued at $15 \mathrm{Km}$ from Fifi, 1우, 26.III.2014 (sweep net).

Distribution. Widespread in Europe, recorded from Russia. First records from Morocco and North Africa.

Hexatoma (Hexatoma) bicolor (Meigen, 1818)

Material examined. Oued at $15 \mathrm{Km}$ from Fifi, $1 \overbrace{}^{\lambda}$, 27.IV.2013, 1ㅇ, 26.III.2014, $3 \hat{\jmath} \widehat{\partial}$, 6.V.2014; Tributary of Oued Ouara, 1ㅇ, 27.IV.2013; Oued Madissouka, 
10, 18.V.2014; Oued Majjou (Hafa meqlouba), 19, 10.V.2014; Aïn Quanquben,

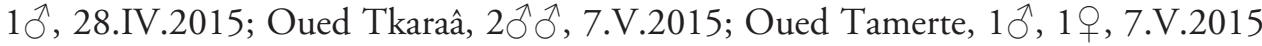
(sweep net).

Distribution. Europe, Georgia, Turkey. First records from Morocco and North Africa.

\section{Pseudolimnophila (Pseudolimnophila) sepium (Verrall, 1886)}

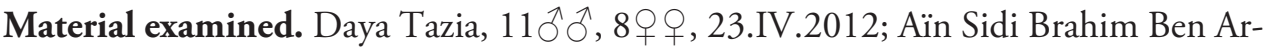
rif (2), 2§ᅫ, 2우, 17.IV.2013; Aïn Afersiw, 1ㅇ, 11.VI.2013; Oued Majjou (Hafa meqlouba), 10, 10.V.2014 (sweep net). Daya Tazia, 1 $\hat{\jmath}, 2$ 우, 14.V.2013; Oued

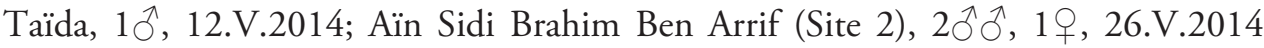
(reared).

Distribution. Europe, Morocco (Rif), Transcaucasia, Turkey, Turkmenistan, Kyrgyzstan, Afghanistan.

\section{Limoniidae: Limoniinae}

\section{Dicranomyia (Dicranomyia) affinis (Schummel, 1829)}

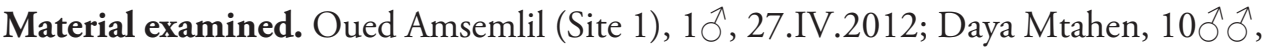
1q, 27.IV.2012; Oued Tkaraâ, 1ठ, 27.IV.2012; Marj El Kheyl, 2우, 30.V.2013;

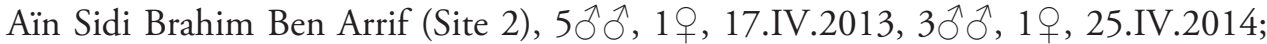

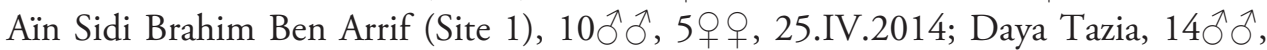

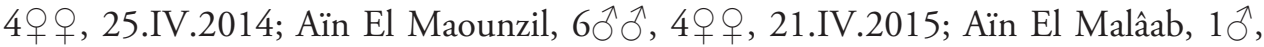

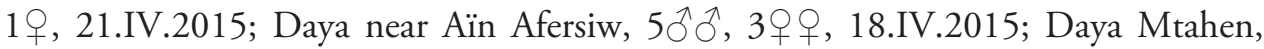
4우, 7.V.2015; Aïn Bab Tariouente, 4ภ̂̉, 28.IV.2015 (sweep net).

Distribution. Great Britain, Ireland, Poland, Morocco (High Atlas).

\section{Dicranomyia (Dicranomyia) chorea (Meigen, 1818)}

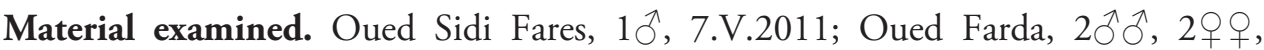

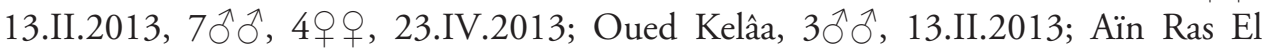
Ma, 10, 3.V.2013; Oued Jnane Niche, $2 \hat{\jmath}$, 2웅, 25.IV.2015; Cascade Chrafate, 10, 2우, 28.IV.2015; Maison forestière, 2웅, 21.IV.2015; Oued El Kanar, 10, 25.IV.2015 (sweep net). Tributary of Oued Zarka, $4 \hat{\jmath} \widehat{\jmath}, 19$, 9.XII.2013 (reared).

Distribution. Nearctic (Canada), Europe, Morocco, Transcaucasia, Turkey, Cyprus, Israel, Iran. First records from the Rif. 


\section{Dicranomyia (Dicranomyia) goritiensis (Mik, 1864)}

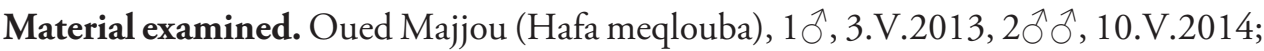
Cascade Chrafate, 1 q, 24.V.2013; Oued El Koub, 1ð , 31.V.2013; Cascade Zarka,

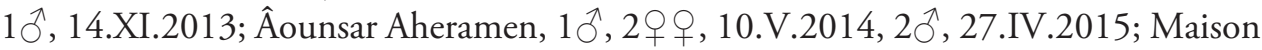
forestière, 10̂, 21.IV.2015 (sweep net).

Distribution. Europe, Morocco, Algeria, Georgia, Turkey, Israel. First records from the Rif.

\section{Dicranomyia (Dicranomyia) longicollis (Macquart, 1846)}

Material examined. Oued Aârate, 4 $\widehat{\partial}$, 3우, 26.III.2014; Barrage Moulay

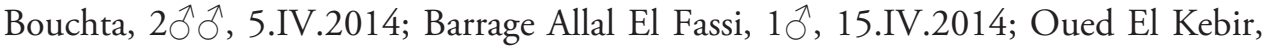
1 , 23.IV.2014 (sweep net).

Distribution. Portugal, Spain, Morocco, Algeria. First records from the Rif and the Middle Atlas.

\section{Dicranomyia (Dicranomyia) modesta (Meigen, 1818)}

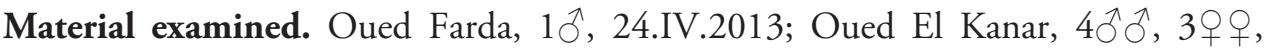

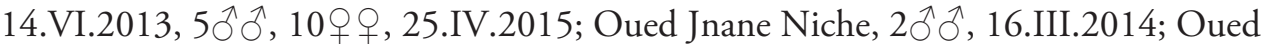
Sidi Yahya Aârab, 4ภํ, 1ㅇ, 25.IV.2015 (sweep net).

Distribution. Nearctic (Canada, USA, Greenland), widespread over Palaearctic, including Central Asia and Mongolia, as far east as Far East of Russia and Japan. First records from Morocco and North Africa.

Dicranomyia (Dicranomyia) novemmaculata (Strobl, 1906)

Material examined. Tributary of Oued el Fondak, 10, 10.II.2013; Oued Aârate, $9 \widehat{\jmath} \hat{0}, 4$ 웅, 26.III.2014 (sweep net).

Distribution. Gibraltar, Portugal, Spain, Algeria. First records from Morocco.

\section{Dicranomyia (Melanolimonia) hamata Becker, 1908}

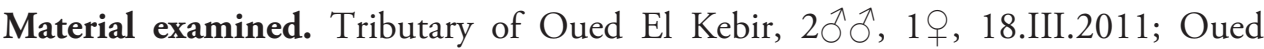

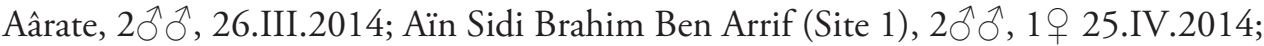
Daya Tazia, 1 $\hat{\jmath}, 25 . I V .2014$ (sweep net).

Distribution. France, Portugal, Spain, Turkey. First records from Morocco and North Africa. 
Dicranomyia (Dicranomyia) ventralis (Schummel, 1829)

Material examined. Lake Badriouen, 2§ึฎ, 26.IV.2011 (sweep net).

Distribution. Europe, Egypt, Azerbaijan, Turkey, Israel, Iran, Russia, Kazakhstan, Kyrgyzstan, Afghanistan, North Korea, India. First records from Morocco and North Africa.

Dicranomyia (Melanolimonia) morio (Fabricius, 1787)

Material examined. Seguia Mtahen, 1ð, 30.V.2013; Daya near Aïn Afersiw, $2 q q$, 11.VI.2013; Daya Jbel Zemzem, 1ð̂, 23.IV.2014 (sweep net).

Distribution. Europe, Morocco, Transcaucasia, Turkey, Iran, Mongolia. First records from the Rif.

\section{Dicranoptycha fuscescens (Schummel, 1829)}

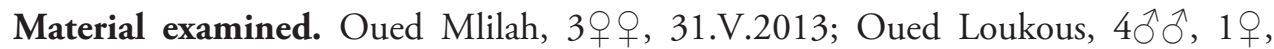
6.V.2015; Oued Zendoula, 19, 6.V.2015 (sweep net).

Distribution. Europe, Morocco, Algeria, Transcaucasia, Turkey, Cyprus, Lebanon, Israel, ?Kazakhstan, Mongolia.

Helius (Helius) bispanicus Lackschewitz, 1928

Material examined. Oued Amsemlil (Site 3), 1, 30.V.2013 (sweep net).

Distribution. Great Britain, Spain, Portugal, Morocco (High Atlas), Georgia, Azerbaijan, Turkey, Cyprus, Iran. First record from the Rif.

Helius (Helius) pallirostris Edwards, 1921

Material examined. Aïn Chouk, 1§̂, 1ㅇ, 25.II.2015 (reared).

Distribution. Europe, Tunisia, Azerbaijan, Israel, Iran, Central Asia. First record from Morocco.

\section{Limonia nubeculosa (Meigen, 1804)}

Material examined. Tributary of Oued El Fondak, 19, 10.II.2013; Aïn Ras El Ma, 1ㅇ, 3.V.2013; Aïn Boughaba, $3 \hat{\jmath} \widehat{\partial}$, 4우, 24.V.2013; Oued Azila, 1ㅇ, 27.VI.2013;

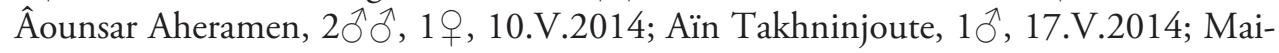


son forestière, 1§, 4우, 17.V.2014; Oued Madissouka, 1 đ, 18.V.2014; Aïn Quanquben, 1 ,, $28 . I V .2015$ (sweep net).

Distribution. Nearctic (Canada, USA), widespread over Palaearctic, including North Africa, Central Asia, and Mongolia, as far east as Far East of Russia, North Korea, and Japan. Recorded from Morocco in the High Atlas. First records from the Rif.

\section{Limonia phragmitidis (Schrank, 1781)}

Material examined. Aïn Quanquben, 1 §, 27.VI.2013 (sweep net).

Distribution. Widespread in the West Palaearctic, also known from Russia, Kazakhstan and Kyrgyzstan. Recorded from Morocco (Middle Atlas). First record from the Rif.

\section{Pediciidae}

\section{Dicranota (Paradicranota) landrocki Czizek, 1931}

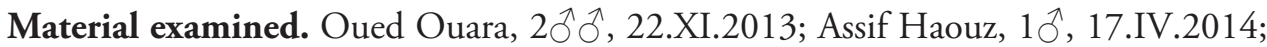

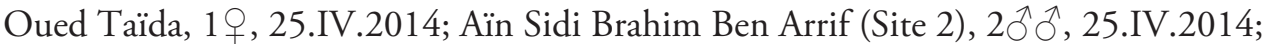
Âounsar Aheramen, 10ิ, 10.V.2014, 10ิ, 27.IV.2015; Oued Tizekhte, 10ิ, 28.II.2015;

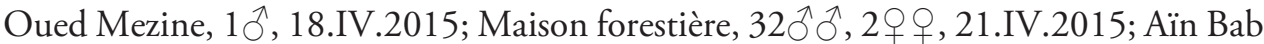
Tariouente, 10ิ, 28.IV.2015 (sweep net).

Distribution. Europe, Russia (North Caucasus), Morocco (Rif), Transcaucasia Lebanon, Tajikistan. First record from the High Atlas.

\section{Dicranota (Ludicia) claripennis (Verrall, 1888)}

Material examined. Oued Amsemlil (Site 1), 1§, 16.XII.2013; Maison forestière, 1ठ, 21.IV.2015 (sweep net).

Distribution. Austria, Belgium, France, Germany, Great Britain, Ireland, Italy, Netherlands, Spain. First records from Morocco and North Africa.

\section{Tricyphona (Tricyphona) immaculata (Meigen, 1804)}

Material examined. Maison forestière, 10, 21.IV.2015; Daya Mtahen, 10, 7.V.2015 (sweep net).

Distribution. Europe, Transcaucasia, Turkey, Lebanon, West Siberia, Central Asia. First records from Morocco and North Africa. 


\section{Discussion}

Altogether a number of 570 specimens, most of which (521) belongs to the Limoniidae distributed in 20 genera, whereas only 49 specimens belong to the Pediciidae distributed in 2 genera.

This study provides an important contribution to the short-palped crane flies fauna of North Africa and Morocco, particularly to the Rif region where 35 species were recorded from the the first time. The most abundant species was Dicranomyia (Dicranomyia) affinis, with 86 specimens, followed successively by, Erioptera (Erioptera) fuscipennis (56), Dicranota (Paradicranota) landrocki (45), Dicranomyia (Dicranomyia) chorea (35), Pseudolimnophila (Pseudolimnophila) sepium (32), Dicranomyia (Dicranomyia) modesta (30) and Molophilus (Molophilus) testaceus (29). The most frequently collected species were Erioptera (Erioptera) fuscipennis (12 sites), Dicranomyia (Dicranomyia) affinis (11 sites), Molophilus (Molophilus) obscurus (10 sites), followed by Dicranomyia (Dicranomyia) chorea, Limonia nubeculosa and Dicranota (Paradicranota) landrocki, all three collected in 9 sites.

18 species of Limoniidae and 2 species of Pediciidae are newly recorded from Morocco, of which 15 species are provided to be new to North Africa. Those new records extend the distribution of the species to most southern localities of the western Palearctics in Africa. Idiocera (Idiocera) ampullifera represents the most southern record (Sahara) of the Limoniidae in Morocco.

Including our results, the total number of species of Limoniidae and Pediciidae known from Morocco now increases from 53 species to 73 (see chekclist).

These new data of crane flies fauna reflect the variety of suitable habitats prospected, such as are springs, mountain rivers and streams, and stagnant water mainly belonging to forest areas. This suggests that many more species and subspecies can yet be found in Morocco, that enjoys a wide variety of geographical and climatic properties.

\section{Check-list of the Moroccan short-palped crane flies}

Limoniidae

Chioneinae

Baeoura ebenina Starý, 1981

Baeoura staryi Driauach \& Belqat, 2015

Cheilotrichia (Empeda) cinerascens (Meigen, 1804)

Cheilotrichia (Empeda) fuscohalterata (Strobl, 1906)*

Cheilotrichia (Empeda) minima (Strobl, 1898)*

Ellipteroides (Ellipteroides) lateralis (Macquart, 1835)

Ellipteroides (Protogonomyia) alboscutellatus (Von Roser, 1840)

Ellipteroides (Protogonomyia) hutsoni (Stary, 1971)

Erioconopa diuturna (Walker, 1848)

Erioconopa symplectoides (Kuntze, 1914) 
Erioptera (Erioptera) fuscipennis Meigen, 1818

Erioptera (Erioptera) lutea lutea Meigen, 1804*

Erioptera (Erioptera) transmarina Bergroth, 1889

Gonomyia (Gonomyia) abscondita Lackschewitz, 1935*

Gonomyia (Gonomyia) subtenella Savchenko, 1972

Gonomyia (Gonomyia) sicula Lackschewitz, 1940*

Gonomyia (Gonomyia) tenella (Meigen, 1818)

Hoplolabis (Parilisia) obtusiapex (Savchenko, 1982)

Hoplolabis (Parilisia) punctigera (Lackschewitz, 1940)

Hoplolabis (Parilisia) sororcula (Lackschewitz, 1940)

Idiocera (Euptilostena) jucunda (Loew, 1873)

Idiocera (Idiocera) ampullifera (Stary, 1979)*

Idiocera (Idiocera) pulchripennis (Loew, 1856)

Idiocera (Idiocera) sziladyi (Lackschewitz, 1940)*

Ilisia maculata (Meigen, 1804)*

Molophilus (Molophilus) ibericus Stary, 2011

Molophilus (Molophilus) obscurus (Meigen, 1818)

Molophilus (Molophilus) propinquus propinquus (Egger, 1863)

Molophilus (Molophilus) testaceus Lackschewitz, 1940*

Symplecta (Symplecta) bybrida (Meigen, 1804)

Symplecta (Trimicra) pilipes (Fabricius, 1787)

Tasiocera (Dasymolophilus) murina (Meigen, 1818)*

Dactylolabinae

Dactylolabis (Dactylolabis) symplectoidea Egger, 1863

Limnophilinae

Austrolimnophila (Austrolimnophila) latistyla Stary, 1977*

Dicranophragma (Brachylimnophila) adjunctum (Walker, 1848)

Dicranophragma (Brachylimnophila) nemorale (Meigen, 1818)

Eloeophila maroccana Stary, 2009

Euphylidorea (Euphylidorea) crocotula (Seguy, 1941)

Euphylidorea (Euphylidorea) dispar (Meigen, 1818)*

Euphylidorea (Euphylidorea) lineola (Meigen, 1804)

Hexatoma (Hexatoma) bicolor (Meigen, 1818)*

Hexatoma (Hexatoma) gaedii (Meigen, 1830)

Pseudolimnophila (Pseudolimnophila) sepium (Verrall, 1886)

Limoniinae

Dicranomyia (Dicranomyia) affinis (Schummel, 1829)

Dicranomyia (Dicranomyia) chorea (Meigen, 1818)

Dicranomyia (Dicranomyia) didyma (Meigen, 1804)

Dicranomyia (Dicranomyia) goritiensis (Mik, 1864)

Dicranomyia (Dicranomyia) longicollis (Macquart, 1846) 
Dicranomyia (Dicranomyia) lutea Meigen

Dicranomyia (Dicranomyia) mitis (Meigen, 1830)

Dicranomyia (Dicranomyia) modesta (Meigen, 1818)*

Dicranomyia (Dicranomyia) novemmaculata (Strobl, 1906)*

Dicranomyia (Dicranomyia) ventralis (Schummel, 1829)*

Dicranomyia (Glochina) sericata (Meigen, 1830)

Dicranomyia (Melanolimonia) hamata Becker, 1908*

Dicranomyia (Melanolimonia) morio (Fabricius, 1787)

Dicranomyia majuscula Pierre, 1924

Dicranoptycha fuscescens (Schummel, 1829)

Geranomyia caloptera (Mik, 1867)

Geranomyia obscura Strobl, 1900

Helius (Helius) hispanicus Lackschewitz, 1928

Helius (Helius) pallirostris Edwards, 1921*

Limonia flavipes (Fabricius, 1787)

Limonia hercegovinae (Strobl, 1898)

Limonia macrostigma (Schummel, 1829)

Limonia nubeculosa Meigen, 1804

Limonia phragmitidis (Schrank, 1781)

Pediciidae

\section{Pediciinae}

Dicranota (Dicranota) bimaculata (Schummel, 1829)

Dicranota (Dicranota) irregularis Pierre, 1922

Dicranota (Paradicranota) candelisequa Stary, 1981

Dicranota (Paradicranota) landrocki Czizek, 1931

Dicranota (Ludicia) claripennis (Verrall, 1888)*

Tricyphona (Tricyphona) immaculata (Meigen, 1804)

\section{Acknowledgements}

The authors sincerely thank Dr. Jaroslav Starý (Olomouc, Czech Republic) for his valuable help concerning the identification of some species.

\section{References}

Driauach O, Belqat B (2015) A new species of the genus Baeoura from Morocco, with a key to the West Palaearctic species (Diptera: Tipuloidea: Limoniidae). ZooKeys 532: 99-105. doi: $10.3897 /$ zookeys.532.5994

Driauach O, Belqat B, De Jong H (2013) A first checklist of the short-palped crane flies (Diptera: Limoniidae, Pediciidae) of Morocco. Boletín de la Sociedad Entomológica Aragonesa 53: $187-190$. 
Eiroa E (2000) Primera cita de Dicranoptycha fusescens (Schummel, 1829) para Marruecos

(Diptera: Limoniidae). Boletín de la Asociación Espanola de Entomología 24: 204.

De Jong H, Oosterbroek P, Gelhaus J, Reusch H, Young C (2008) Global biodiversity of craneflies (Insecta, Diptera:Tipulidea or Tipulidae sensu lato) in freshwater. Hydrobiologia 595: 457-467. doi: 10.1007/s10750-007-9131-0

Oosterbroek P (2015) Catalogue of the Craneflies of the World (Insecta, Diptera, Nematocera,

Tipuloidea). http://ccw.naturalis.nl/index.php [version 26 November 2015]

Pârvu C, Rãzvan PM, Rãzvan Z (2006) Faunistic data on some dipteran families (Insecta: Diptera)

from Morocco. Travaux du Muséum d'Histoire Naturelle "Grigore Antipa” 49: 271-281.

Pierre C (1922a) Nematocera Polyneura recueillis au Maroc par M. Charles Alluaud (1919-

1920) (Insectes Diptères). Bulletin de la Société des Sciences naturelles du Maroc 1: 21-24.

Pierre C (1922b) Nematocera Polyneura recueillis au Maroc par M. Charles Alluaud (2e liste,

(1920-1921) (Insectes Diptères). Bulletin de la Société des Sciences naturelles du Maroc 1: $148-151$.

Pierre C (1924) Nematocera Polyneura receuillis au Maroc par M. Charles Alluaud (3e liste, 1922

1923) (Insectes Diptères). Bulletin de la Société des Sciences naturelles du Maroc 4: 198-201.

Reusch H, Oosterbroek P (1997) Diptera Limoniidae and Pediciidae, Short-palped Crane

Flies. In: Nilsson A (Ed.) Aquatic Insects of North Europe 2: 105-132.

Savchenko EN, Oosterbroek P, Starý J (1992) Family Limoniidae. Catalogue of Palaearctic Diptera 1: 183-369.

Séguy E (1941a) Récoltes de R. Paulian et A. Villiers dans le haut Atlas marocain, 1938, (XVIIe note) Diptères. Revue Française d'Entomologie 8: 25-33.

Séguy E (1941b) Diptères receuillis par M. Berland dans le sud marocain. Annales de la Société Entomologique de France 110: 1-23.

Starý J (1971) A new palaearctic representative of the subgenus Protogonomyia Alexander (Diptera, Tipulidae). Acta Entomologica Bohemoslovaca 68: 319-321.

Starý J (1974) The identity of Gonomyia (Idiocera) sexguttata (Diptera, Tipulidae). Acta Entomologica Bohemoslovaca 71: 136-140.

Starý J (2006) Hoplolabis (Parilisia) species related to $H$. (P.) punctigera (Lackschewitz, 1940) and $H$. (P.) spinosa (Nielsen, 1953) with the description of a new species (Diptera,Limoniidae). Studia dipterologica 13: 115-125.

Starý J (2009) West Palaearctic species of the genus Eloeophila (Diptera: Limoniidae). European Journal of Entomology 106: 425-440. doi: 10.14411/eje.2009.054

Starý J (2011) Descriptions and records of the Palaearctic Molophilus Curtis (Diptera, Limoniidae). Zootaxa 2999: 45-62.

Starý J, Kubik S, Bartak M (2005) Limoniidae. In: Bartak M, Kubik S (Eds) Diptera of Podyji National Park and its environs. Ceska Zemedelska Univerzita v Praze, 24-32.

Starý J, Oosterbroek P (2008) New records of West Palaearctic Limoniidae, Pediciidae and Cylindrotomidae (Diptera) from the collections of the Zoological Museum, Amsterdam. Zootaxa 1922: 1-20.

Vaillant F (1956) Recherches sur la faune madicole (Hygropetriques.l.) de France, de Corse et d'Afrique de Nord. Mémoires du Museum National d'Histoire Naturelle (N.S.), Série A, Zoologie 11: 1-257. 